\title{
Investigation of Epithelial-to-Mesenchymal Transition through Microcontact Printing
}

\author{
Huma Inayat \\ Graduate program in Biomedical Engineering \\ Ryerson University \\ Toronto, Canada \\ inayathu@ryerson.ca \\ Scott S. H. Tsai* \\ Department of Mechanical and Industrial engineering \\ Ryerson University \\ Toronto, Canada \\ scott.tsai@ryerson.ca \\ Andras Kapus* \\ Keenan Research Centre for Biomedical Science \\ St. Michael's Hospital \\ Toronto, Canada \\ kapusA@smh.ca \\ *Co-corresponding authors.
}

\begin{abstract}
We have used microcontact printing as a means of simulating injury or wounds in a healthy monolayer of LLC PK1 cells to study the expression of Epithelial-to-Mesenchymal Transition (EMT) signature proteins. Cells were patterned on PDMS substrates using stamps that were prepared in the cleanroom using conventional soft lithography techniques. Patterned cells were exposed to an external stimulus of Transformation Growth Factor $\beta$ (TGF $\beta$ ) and analyzed further for the presence and upregulation of EMT signature proteins that are indicative of the progression of EMT.
\end{abstract}

Microcontact Printing, Epithelial-to-Mesenchymal Transition, LLC PK1 cells, TGFB

\section{Introduction}

Organ fibrosis is considered a failed or dysregulated wound healing process, triggered by prolonged and repetitive injury to epithelium and endothelium. It was first discovered in 1995, that fibroblasts that contribute to fibrosis often originate from the local conversion of epithelium. ${ }^{1}$ This discovery set forward a slurry of research activity around the phenomenon of Epithelialto-Mesenchymal transition or EMT, and its role in organ fibrosis. ${ }^{2}$ EMT is a process through which epithelial cells lose their strong intercellular contacts and polarity, and transition to migratory and often invasive mesenchymal cell types. ${ }^{3}$ These mesenchymal cells then mediate either physiological functions (e.g. tissue differentiation during embryogenesis or normal wound healing), or participate in pathological processes (fibrosis, cancer). ${ }^{3}$

Our goal is to investigate the concept that epithelial wounding or injury (which can be simulated in vitro by the absence of cell-cell contacts), predisposes the injured cells for various features of EMT, including the expression of EMT signature proteins (such as alpha-Smooth Muscle Actin). We also aim to investigate the proportional secretory response (termed profibrotic epithelial phenotype or PEP) caused by the loss of cell-cell contacts (or injury) which holds true even if EMT is just partial. We hypothesize that the length of free epithelial edges in a monolayer is proportional to the propensity of the epithelium to acquire a profibrotic epithelial phenotype.

To address this hypothesis, we have used microfabrication to establish a model in which the proportionality of the response can be investigated. To establish our gold standard, initially we investigated SMA expression., the hallmark of full-blown EMT. The next step will be the investigation of the secretory phenotype. 


\section{EXPERIMENTAL SETUP}

\section{A. Cell Culture and treatment}

We use porcine kidney proximal tubule cell line (LLC-PK1) used to conduct all experiments as described in previous studies. ${ }^{4}$ Cells are cultured using DMEM supplemented with $10 \%$ fetal bovine serum and $1 \%$ penicillin/streptomycin. After patterning, cells are treated with $5 \mathrm{ng} / \mathrm{ml} \mathrm{TGF} \beta$ in serum free medium for 48 hours. Cells are washed with phosphate- buffered saline (PBS) prior to analysis.

\section{B. Mold fabrication for Microcontact printing}

The stamps used for patterning cells are prepared using conventional soft lithography techniques in a cleanroom. ${ }^{5}$ Stamps are incubated with $80 \mathrm{ug} / \mathrm{ml}$ Fibronectin prior to stamping. Polydimethylsiloxane (PDMS) is used to line 6-well plates to prepare surface for cell patterning.

\section{REFERENCES}

1. Strutz, F. et al. Identification and characterization of a fibroblast marker: FSP1. J. Cell Biol. 130, 393-405 (1995).

2. Quaggin, S. E. \& Kapus, A. Scar wars: mapping the fate of epithelial-mesenchymal-myofibroblast transition. Kidney Int. 80, 41-50 (2011).

3. Thiery, J. P., Acloque, H., Huang, R. Y. J. \& Nieto, M. A. Epithelial-Mesenchymal Transitions in Development and Disease. Cell 139, 871-890 (2009).

4. Kapus, A. et al. Differential Topical Susceptibility to TGF $\beta$ in Intact and Injured Regions of the Epithelium: Key Role in Myofibroblast Transition. Molecular Biology of the Cell 21, 3326-3336 (2013).

5. Whitesides, G.M. et al. Soft Lithography. Annual review of Materials Science 28, 153-184. (1998) 